\title{
Henry James en France et en Europe: les recherches, les traductions, l'enseignement et les sentiments. Une interview avec Mme le Professeur Annick Duperray
}

DOI: http://dx.doi.org/10.12775/LC.2017.015

Grzegorz Koneczniak: Madame le Professeur, je vous remercie de bien vouloir répondre à mes questions concernant Henry James et son ouvre. La première question: Pouvez-vous décrire votre expérience dans le rôle de Directrice de la Société the European Society of Jamesian Studies (ESJS)?

Annick Duperray: La Société (ESJS) existe depuis le mois d'avril 2009 et s'est constituée lors d'une assemblée générale à l'occasion d'un premier colloque organisé à Paris (The American University of Paris /AUP) les 3 et 4 avril 2009. Sa mission est de contribuer à la promotion des études jamesiennes en Europe, tout en maintenant contacts et relations avec d'autres organismes, notamment la société transatlantique (The Henry James Society).

Comment concevez-vous votre travail dans la Société? Quels sont vos objectifs à propos des recherches sur James et ses ouvres?

En tant qu'association, la Société s'est dotée d'un bureau constitué de 3 personnes qui exercent surtout un rôle symbolique et visent à mettre en relation les chercheurs tout en suscitant des projets et rencontres. Ces projets ont jusqu'à ce jour pris la forme de colloques internationaux organisés par des spécialistes de l'œuvre de James et pris en charge par les universités d'accueil. Chaque colloque a donné lieu à publication sélective. Deux colloques ont été organisés à ce jour à l'Université Américaine de Paris (AUP): «Henry James's Europe, Heritage and Transfer» (2009), et «Henry James and the Poetics of Duplicity» (2010). Le premier colloque international organisé en Pologne en 2013 à propos d'Henry James («Henry James: His Great Wars and Battles») a été aussi l'occasion d'une collaboration scientifique fructueuse entre les chercheurs polonais de l'Université Nicolas Copernic de Toruń et la ESJS. Enfin c'est à nouveau à l'Université Américaine de Paris (AUP) qui se tiendra, du 20 au 22 octobre 2016, à l'occasion du centenaire de la mort de l'auteur, et 
à l'initiative de la ESJS, un troisième colloque international: «Reading Henry James in the Twenty-First Century: Heritage and Transmission».

Et quelles sont vos prévisions sur les activités de la Société dans l'avenir?

L'avenir de la Société dépendra de manière très pragmatique des propositions faites par des chercheurs dans le cadre de leurs activités universitaires. Toutes les initiatives privilégiant la dimension européenne de l'œuvre de James, ainsi que les interrelations entre texte et théorie littéraire, seront bienvenues.

Les ouvres de James. Parmi ses textes littéraires lequel trouvez-vous le plus difficile et pourquoi?

Les œuvres de la phase majeure sont aussi les plus complexes, notamment Les ailes de la colombe, où l'art de l'ambiguïté est porté à l'extrême.

Et maintenant les sentiments. Madame le Professeur, vous avez édité les volumes des Nouvelles complètes d'Henry James. Lequel de ses textes vous a le plus émue?

Le choix est difficile - «L'élève» probablement, et la manière dont la «vision brouillée» du jeune protagoniste constitue le véritable apprentissage de la vérité.

Et vos étudiants. Pouvez-vous partager avec nous votre expérience dans l'enseignement et décrire la réception des textes écrits par James et lus par vos étudiants?

Les nouvelles ou les romans courts demeurent pour des raisons évidentes les plus accessibles. «Ce que savait Maisie» et «Le tour d'écrou» ont souvent figuré dans les programmes de littérature en troisième année (licence).

Comment évaluez-vous l'importance de James dans les cours de littérature américaine en France de nos jours?

L'accent porté actuellement sur la dimension culturelle («cultural studies») de l'œuvre littéraire n'est pas toujours très propice à l'étude d'auteurs tels qu'Henry James dont l'impact repose sur l'intensité esthétique de la prose.

Et maintenant une question similaire. Madame le Professeur, vous avez publié Echec et écriture: essai sur les nouvelles de Henry James (1993) et The reception of Henry James in Europe (2006). Vous traitez aussi de textes écrits par Paul Auster, Nathaniel Hawthorne et Flannery O'Connor dans vos publications. Comment pourriez-vous décrire les recherches sur James et ses œuvres faites en France et en Europe? Quelles sont les perspectives populaires et lesquels des textes écrits par James sont interprétés le plus souvent.

Tous ces auteurs, au-delà des disparités évidentes dans les sujets abordés et les modes d'écriture, ont en commun le rapport étroit entre éthique et esthétique, «C'est l'art qui fait la vie», pour reprendre la célèbre formule d'Henry James.

Comment pourriez-vous décrire les recherches sur James et ses œuvres en France et en Europe? 
A partir de 1960 le développement des théories du langage et de l'analyse textuelle fut très propice, en Europe (notamment en France et en Italie), à la lecture et à la traduction d'auteurs comme Henry James, malgré les excès d’approches formalistes trop radicales.

Comment évaluez-vous les traductions des textes de James en France? Quelle est votre expérience de la traduction? Qu'est-ce qui est plus difficile: traduire les nouvelles (les textes plus courts) ou les romans (les textes plus longs) de James?

La difficulté posée par la traduction n'est pas toujours tributaire de la longueur du récit. Des nouvelles comme «Le tour d'écrou» («The Turn of the Screw») ou «Le coin plaisant» («The Jolly Corner») posent des problèmes tout aussi épineux que les grands romans de la phase majeure. En France on peut considérer que la totalité des romans et nouvelles de James ont été traduits, sans oublier l'autobiographie, les carnets et une large sélection de ses essais et récits de voyage. Deux éditeurs - les éditions Gallimard / collection de la Pléiade, ainsi que les éditions de La Différence - ont publié des traductions de l'intégralité des nouvelles. L'édition de la Pléiade, co-dirigée par Evelytne Labbé et moi-même, offre pour chaque récit, comme le pratique toujours la Pléiade, un appareil critique complet, (introduction, notes et notices). La Pléiade vient de publier (en 2016), sous la direction d'Evelyne Labbé, un premier volume rassemblant plusieurs romans de James. De telles initiatives sont évidemment propres à maintenir, et surtout développer, l'intérêt des lecteurs pour un auteur réputé difficile.

\section{Dans le domaine de la traduction, que pourriez-vous entreprendre à l'avenir pour} rendre les œuvres de James plus populaires en France?

Il existe déjà des rééditions de traductions dans des collections «grand public», comme Gallimard Folio Classique - collection qui vient de rééditer, sous ma direction et à l'occasion du centenaire de la mort de James, la traduction annotée et commentée de la première édition américaine des Carnets - celle qui fut entreprise par F.O. Matthiessen et Kenneth Murdock et publiée en 1947. Ces rééditions grand public sont propices, cela va de soi, à la popularisation des œuvres de James.

Je crois qu'il y a quelques adaptations de James pour le cinéma ou pour la télévision en France. Pouvez-vous les décrire?

La production française est surtout faite de téléfilms. De 1974 à 2007 on compte - principalement et parmi les plus connus - 9 téléfilms et seulement trois longs métrages, à savoir, en 1978: La Chambre verte, film réalisé par François Truffaut d'après trois nouvelles: «L'Autel des morts», «La Bête dans la jungle» et «Les Amis des amis»; en 1981 Les Ailes de la colombe de Benoît Jacquot; et enfin en 1996 L'Élève, réalisé par Olivier Schatzky, d’après la nouvelle éponyme. Mentionnons de surcroît Aspern, film franco-portugais d'Eduardo de Gregorio. Il y a une raison à cette spécificité française. Pour les cinéastes de la Nouvelle Vague comme Jacques Rivette ou François Truffaut, une adaptation filmique demeure fondamentalement trop littérale et ne permet pas de restituer le caractère énigmatique et la dimension d'indicible propres à l'écriture d'Henry James. La chambre verte n'en demeure pas moins, sur le plan international, l'une des meilleures restitutions filmiques des spécificités et ambiguités de l'humeur jamesienne. Il est frappant d'ailleurs de remarquer à quel point les restitutions les plus efficaces et suggestives sont parfois les plus indirectes et les plus
Henry James and the

Poetics of Duplicity

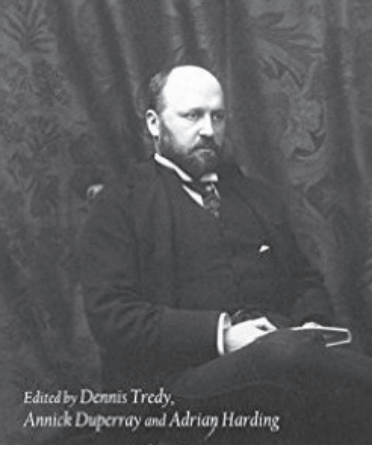

Edird by Dernis Tredy.

Annick Duparray and Adriag Harding 
inattendues, les plus «atmosphériques» pour reprendre un adjectif cher à James. On citera par exemple dans cette optique Céline et Julie vont en bateau (1974) de Jacques Rivette luimême, lointainement inspiré de deux œuvres de James. Plus récemment, le film de Patrice Leconte sorti en 2004 et intitulé Confidences trop intimes réussit à restituer une humeur jamesienne au-delà d'une référence, apparemment anodine et accessoire, à la nouvelle intitulée «La bête dans la jungle». Notons que l'efficacité de ce non-dit n’a pas échappé à Leo Bersani et Adam Phillips: en effet leur ouvrage - Intimacies (2006) - débute par une analyse subtile du film de Patrice Leconte et de la manière dont cet intertexte apparemment fortuit et accessoire va, à mesure que se déroule l'intrigue, produire du sens.

Merci pour vos réponses, Madame le Professeur.

Interview par Grzegorz Koneczniak

* Ph.D., il travaille au Département d'anglais de I'Université Nicolas Copernic de Toruń. Ses recherches portent sur la littérature irlandaise et canadienne.E-mail: gregorex@umk.pl. 